\title{
A New Luciferase Reporter Gene Assay for the Detection of Androgenic and Antiandrogenic Effects Based on a Human Prostate Specific Antigen Promoter and PC3/AR Human Prostate Cancer Cells
}

\author{
Ryoichi KIzU, ${ }^{* 1, * 2 \uparrow}$ Naoki OTsuki, ${ }^{33}$ Yoshiko Kishida, ${ }^{* 1}$ Akira Toriba, ${ }^{* 3}$ Atsushi Mizokami,*4 \\ Kerry L. Burnstein, ${ }^{* 5}$ Carolyn M. KLInge, $* 6$ and Kazuichi HayaKawa*1,*2 \\ *1 Graduate School of Natural Science and Technology, Kanazawa University, Kanazawa 920-0934, Japan \\ *2 Core Research for Evolutional Science and Technology (CREST), \\ Japan Science and Technology Corporation, Kawaguchi 332-0012, Japan \\ *3 Faculty of Pharmaceutical Sciences, Kanazawa University, Kanazawa 920-0934, Japan \\ *4 Department of Urology, School of Medicine, Kanazawa University, Kanazawa 920-8641, Japan \\ *5 Department of Molecular and Cellular Pharmacology, University of Miami School of Medicine, \\ Miami, Florida 33101, USA \\ *6 Department of Biochemistry and Molecular Biology, University of Louisville School of Medicine, \\ Louisville, Kentucky 40292, USA
}

\begin{abstract}
We developed a new mammalian cell-based luciferase reporter gene assay for androgenic and antiandrogenic activities of chemicals and environmental samples. Environmental samples usually have a complex matrix that may contain the constituents acting as androgen receptor (AR) agonists, AR antagonists or aryl hydrocarbon receptor (AhR) agonists. AhR agonists are known to elicit the antiandrogenic effect through cross-talk between AR and AhR signal transduction pathways. In this study, PC3/AR human prostate carcinoma cells were transiently transfected with a prostate-specific antigen (PSA) promoter-driven luciferase expression plasmid. The cells were treated with a test compound or an environmental sample for $24 \mathrm{~h}$ at $37^{\circ} \mathrm{C}$ and then measured for luciferase activity. The luciferase activity was induced by dihydrotestosterone (DHT) in a concentration-dependent manner in a concentration range from $10 \mathrm{fM}$ to $1 \mathrm{nM}$. R1881, a synthetic androgen receptor agonist, induced luciferase activity and its inductive effects was additive to that of DHT. The luciferase activity was not induced by cortisol, a glucocorticoid, progesterone, a progestin, and $17 \beta$-estradiol, an estrogen in a concentration range of up to $1 \mu \mathrm{M}$. DHT-induced luciferase activity was reduced by bicalutamide and cyproterone acetate, AR antagonists, and also by benzo[a]pyrene, an aryl hydrocarbon receptor agonist, through AhR-mediated pathways. All of these findings indicate that the present assay system correctly responds to AR agonists, AR antagonists and AhR agonist and, therefore, it is a powerful tool for the sensitive and selective screening of chemicals and environmental samples for their androgenic and antiandrogenic activities. We developed the first assay system, in which the expression of luciferase was driven by the promoter of a prostate-specific antigen gene, a typical human androgenregulated gene.
\end{abstract}

(Received September 30, 2003; Accepted November 10, 2003)

\section{Introduction}

In recent years, there has been increasing concern that man- and nature-made chemicals may cause the dysfunction of human and wildlife endocrine systems, leading to adverse health effects, including increased rates of certain hormone-related cancers, such as breast and prostate, reproductive system abnormalities, immune system deficiencies and declines in wildlife populations. ${ }^{1-3}$ Such chemicals have been classified as endocrine disruptors. Endocrine disruptors may be producing

\footnotetext{
† To whom correspondence should be addressed.

E-mail: kizu@p.kanazawa-u.ac.jp
}

adverse effects in humans and wild life by directly or indirectly disrupting the endocrine system through mimicking or antagonizing natural hormones. While a wide variety of chemicals, including environmental pollutants, industrial chemicals and natural products, have been studied concerning their effects on endocrine functions, most of these studies have dealt with the estrogenic and antiestrogenic activities of substances.

The number of environmental chemicals identified with antiandrogenic properties is increasing. ${ }^{4}$ There have also been several studies indicating that certain chemicals can impair the function and development of male reproductive systems. ${ }^{4}$ Mice exposed in utero to hydroxyflutamide, an antiandrogen, showed an inability to impregnate. ${ }^{5}$ A dysfunction of the male 
reproductive system was found in alligators in Lake Apopka, Fl, USA, which is heavily polluted with DDT and its metabolites. ${ }^{6}$ There have been studies reporting a global decline in semen quality (sperm density) of humans during the past 50 years. ${ }^{7,8}$ Androgens have a pivotal role in the development and maintenance of the male reproductive system. ${ }^{9,10}$ To ensure the safety of chemicals and the environment, it is important to obtain information about the androgenic and antiandrogenic activities of chemicals and environmental samples.

Natural hormones and endocrine-modulating chemicals elicit their effects through the transcriptional activation of hormoneresponsive genes. Thus, the reporter gene assay technique may be suitable for detecting the hormonal activities of chemicals and environmental samples because it has been developed to detect the enhancer and promoter activities of genes. Several mammalian cell-based reporter gene assay systems have been developed for the in vitro evaluation of androgenic and antiandrogenic activities of chemicals. ${ }^{1-15}$ All of these systems adopted the mouse mammary tumor virus (MMTV) promoter for transcriptional activation of the reporter gene. There is no assay system in which the transcription of the reporter gene is driven by enhancer/promoters of human androgen-regulated genes. On the other hand, it has been reported that chemicals with aryl hydrocarbon receptor (AhR) agonist activity, such as 2,3,7,8-tetrachlorodibenzo- $p$-dioxin (2,3,7,8-TCDD) and some polycyclic aromatic hydrocarbons (PAHs) having four or more rings, shows antiandrogenic effects through AhR-mediated mechanisms without binding to AR. ${ }^{16-18}$ There is also no assay system which responds correctly to AhR agonists. Dioxins and polycyclic aromatic hydrocarbons are ubiquitous environmental pollutants. Taking into consideration that environmental samples mostly involve constituents acting as an AhR agonist, it is necessary to develop an assay method that responds correctly to androgen receptor (AR) agonists, AR antagonist and AhR agonists.

We report here on a new luciferase reporter gene assay for the androgenic and antiandrogenic activities of chemicals and environmental samples. In this study, we used two androgenregulated luciferase expression plasmid vectors, pGLPSAp5.8 ${ }^{19}$ and pMMTV-Luc, ${ }^{20}$ and two human-originated cell lines, PC3/AR ${ }^{21}$ and T47D, in combinations. The combination of PC3/AR cells and pGLPSAp5.8 vector was found to the most suitable, responding sensitively and selectively to AR agonists, $\mathrm{AR}$ antagonists and AhR agonists.

\section{Experimental}

\section{Chemicals}

Dihydrotestosterone (DHT), cortisol (C) and progesterone (P) and $17 \beta$-estradiol $\left(\mathrm{E}_{2}\right)$ of biochemical study grade, anthracene (Ant), benzo[k]fluoranthene $(\mathrm{BkF})$, benzo[a]pyrene $(\mathrm{BaP})$ and pyrene (Pyr) of environmental analysis standard grade, and ethanol of ultrapure grade were purchased from Wako Pure Chemicals (Tokyo, Japan). R1881 (methyl trienolone) was purchased from Daiichi Pure Chemicals (Tokyo, Japan). $\alpha$ Naphthoflavone $(\alpha-\mathrm{NF})$ and cyproterone acetate (CPA) was from Sigma (St. Louis, MO, USA). Bicalutamide (BCT; Casodex) was a gift from AstraZeneca (Cheshire, UK). All other chemicals were of reagent grade or better from commercial sources and were used as received. Chemicals used for cell treatment were all dissolved in $50 \%(\mathrm{v} / \mathrm{v})$ ethanol.

\section{Cell culture}

A human prostate carcinoma cell line PC3/AR was established by Burnstein et al. ${ }^{21}$ Human breast carcinoma T47D cells were purchased from Dainippon Pharmaceutical (Tokyo, Japan). PC3/AR and T47D cells were cultured at $37^{\circ} \mathrm{C}$ in a humidified atmosphere of $5 \% \quad \mathrm{CO}_{2}-95 \%$ air. In routine maintenance, the cells were grown in phenol red-free RPMI1640 medium supplemented with $10 \%$ fetal bovine serum (FBS) (Sigma), $100 \mu \mathrm{g} / \mathrm{ml}$ streptomycin, $10 \mathrm{units} / \mathrm{ml}$ penicillin, and passaged with trypsinization every fourth day. In assays, cells were cultured in an assay medium of phenol red-free RPMI1640 medium supplemented with $5 \%$ charcoal/dextran-treated FBS (Hyclone; Logan, Utah, USA), $100 \mu \mathrm{g} / \mathrm{ml}$ streptomycin and 10 units/ml penicillin.

\section{Luciferase reporter assay}

PC3/AR or T47D cells $\left(5 \times 10^{6}\right.$ cells $)$ were harvested, and washed once with cold phosphate-buffered saline. The cells were suspended in $5 \mathrm{~mL}$ of transfection medium of FBS-free OPTI-MEM I medium (GIBCO) containing $20 \mu \mathrm{g}$ of a luciferase reporter vector and $50 \mu \mathrm{L}$ of LipofectAMINE (GIBCO) and transiently transfected with a luciferase expressing plasmid vector for $30 \mathrm{~min}$ at $37^{\circ} \mathrm{C}$. The vector transfected to the cells was pGLPSAp5.8, pMMTV-Luc or pGL3-control vector (Promega, Madison, WI, USA). Then, 25 $\mathrm{mL}$ of the assay medium was added to the cell-suspension solution and the cells were plated on 48 -well plates at a cell density of $5 \times 10^{4}$ cells per well $(300 \mu \mathrm{L}$ of diluted cell suspension solution per well). After $24 \mathrm{~h}$, the cells were washed once with fresh assay medium and treated with $0.2 \%(\mathrm{v} / \mathrm{v})$ ethanol (blank), DHT, R1881, C, P, E 2, BCT, CPA, BaP or $\alpha$ $\mathrm{NF}$ alone or in combinations for $24 \mathrm{~h}$. The final ethanol concentration in the medium was adjusted at $0.2 \%(\mathrm{v} / \mathrm{v})$. After the treatment, the cells were lysed with $50 \mu \mathrm{L}$ of PicaGene cell lysis buffer LUC (Toyo Ink; Tokyo, Japan). The luciferase activity in a cell lysate was measured using a PicaGene luciferase kit (Toyo Ink) according to the manufacture's protocol and normalized to the protein concentration measured by a protein assay kit (Bio-Rad; Hercules, CA, USA).

\section{Statistical analysis}

Statistical analyses were performed using an unpaired Student's $t$-test with StatView-J 5.0 for a Macintosh computer (Nankodo; Tokyo, Japan). A value of $p<0.05$ was considered to be significant.

\section{Results and Discussion}

The MMTV promoter has been used in mammalian cell-based reporter gene assays developed for the in vitro evaluation of the androgenic and antiandrogenic activities of chemicals. ${ }^{11-15}$ It hardly needs to be said that the luciferase expression system driven by a promoter of human androgen-regulated gene is more desirable. Another important problem is the responsiveness to AhR agonists. Typical AhR agonists, such as 2,3,7,8-TCDD and certain PAHs, are found to elicit antiandrogenic effects through AhR-mediated mechanisms without binding to AR. ${ }^{16-18}$ We demonstrated that diesel exhaust particle extracts exhibited antiandrogenic effects in PC3/AR cells, and that the major part of the antiandrogenic effects are caused by constituents with AhR agonist activity. ${ }^{22}$ Further, the importance of the AhR agonist on male reproductive-system functions has been suggested by in vivo studies. It has been shown that 2,3,7,8-TCDD impairs male reproductive functions in animal models ${ }^{4}$ and that TCDD exerts its adverse effects without affecting the circulatory androgen 

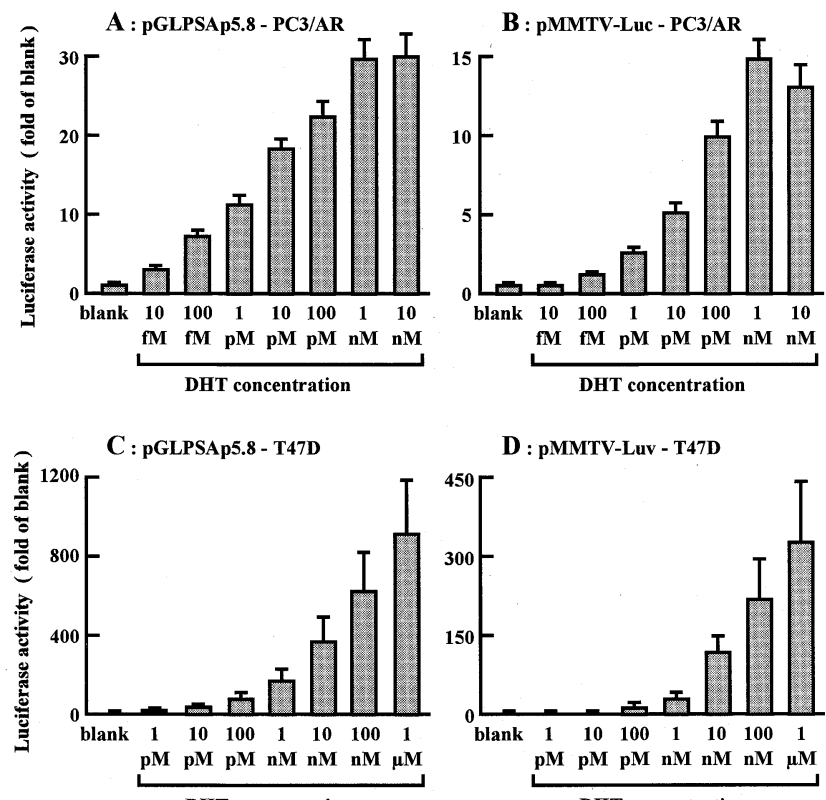

D : pMMTV-Luv - T47D

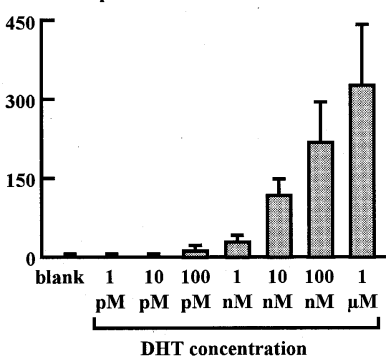

Fig. 1 Responsiveness of the four androgen-responsive luciferase expressing systems to DHT. Each column and vertical bar represent the mean and SD, respectively, from five separate cultures.

levels and steroidogenic enzyme activities in rats, ${ }^{23-25}$ suggesting that activated AhR is implicated in the TCDD-induced dysfunction of the male reproductive system. An assay system has to respond not only to AR agonists and antagonists, but also to AhR agonists in order to screen chemicals and environmental samples for their androgenic and antiandrogenic effects.

The prostate specific antigen (PSA) gene is a typical androgen-regulated gene expressed in the prostate gland, an accessory sex organ. In our previous study, we found that PAHs with AhR agonist activity antagonized the androgeninduced expression of genomic PSA. ${ }^{18}$ The PSA promoter was considered to be promising for developing a new assay system. In this study, we used a pGLPSAp5.8 plasmid vector constructed by Mizokami et al., ${ }^{19}$ in which a 5.8 kilo bases region of the PSA promoter was cloned to drive the expression of the luciferase gene in response to the androgen action.

On the other hand, cell lines expressing wild-type human AR are few. In this study, we used a human prostate carcinoma cell line, PC3/AR, and a human breast carcinoma cell line, T47D. PC3/AR, established by Burnstein et al. ${ }^{21}$ was derived from a human prostate carcinoma cell line, PC-3, by transforming to stably express human wild-type AR.

In this study, the responsiveness of the pGLPSAp5.8-PC3/AR and pGLPSAp5.8-T47D systems to AR agonists, AR antagonists and AhR agonists was evaluated and compared with those of the MMTV-PC3/AR and MMTV-T47D systems.

Responsiveness of the four luciferase expressing systems to DHT and other hormones

First, we evaluated the responsiveness of the four luciferase expressing systems to DHT, a natural androgen. The luciferase activity increased with an increase in the DHT concentration in the four systems, as shown in Fig. 1. Significant differences were observed between the PC3/AR and T47D cell-based systems. While the T47D systems yielded higher luciferase activity (expressed as fold of blank) than the PC3/AR systems on both the pGLPSAp5.8 and pMMTV-Luc vectors, the

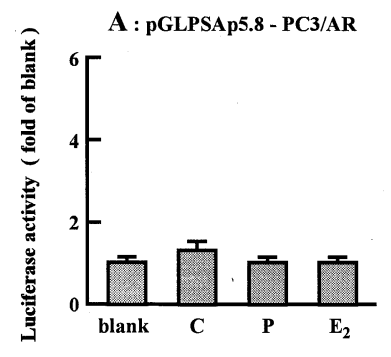

B : pMMTV-Luc - PC3/AR
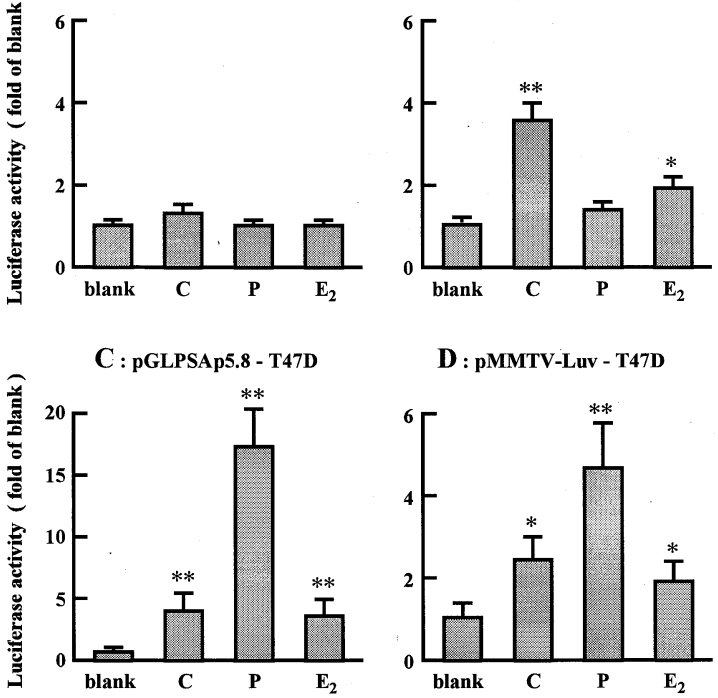

D : pMMTV-Luv - T47D

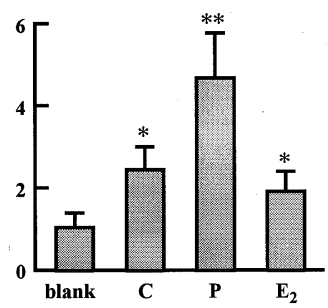

Fig. 2 Responsiveness of the four androgen-responsive luciferase expressing systems to $\mathrm{C}, \mathrm{P}$ or $\mathrm{E}_{2}$. Concentration of $\mathrm{C}, \mathrm{P}$ and $\mathrm{E}_{2}$ : 100 nM. Each column and vertical bar represent the mean and SD, respectively, from five separate cultures. $*$ and $* *$ : Significantly different from blank ( $p<0.05$ and $p<0.01$, respectively).

reproducibility of luciferase activity was larger in the T47D systems than in the PC3/AR systems. The relative standard deviations were approximately $35 \%$ in the T47D systems and less than $10 \%$ in the PC3/AR systems. On the other hand, the PC3/AR systems induced luciferase at a lower DHT concentration than in the T47D systems. The minimal DHT concentration to induce luciferase was $10 \mathrm{fM}$ on the PC3/AR systems and $10 \mathrm{nM}$ on the T47D systems. Taking into consideration the variation of the luciferase activity and DHT concentration at which luciferase was induced, the PC3/AR cell systems is preferable to the T47D systems. Comparing the pGLPSAp5.8 and pMMTV-Luc vectors, the pGLPSAp5.8 vector gave an approximately two-times higher luciferase activity than the pMMTV-Luc vector in both PC3/AR and T47D cells.

Since the consensus sequences of glucocorticoid- and progestin-response elements are identical to that of the androgen-response element, ${ }^{26} \mathrm{PC}-3$, the parent cell line of the PC3/AR, and T47D cell lines are known to express other endogenous receptors than AR: glucocorticoid receptor in PC $-3^{27}$ and progesterone receptor in T47D. ${ }^{28}$ Thus, the responsiveness of the four systems to other hormones was examined. The hormones used were $\mathrm{C}$, a glucocorticoid; $\mathrm{P}$, a progestin; and $\mathrm{E}_{2}$, an estrogen. The obtained results are shown in Fig. 2. In the PC3/AR systems, luciferase was induced by $\mathrm{C}$ $(100 \mathrm{nM})$, but the effect of $\mathrm{C}$ was not statistically significant in the pGLPSAp5.8-PC3/AR system. In the pMMTVLuc-PC3/AR system, luciferase was also induced by $\mathrm{E}_{2}(100$ $\mathrm{nM})$. In the T47D systems, luciferase was induced by $\mathrm{C}(100$ $\mathrm{nM}), \mathrm{P}(100 \mathrm{nM})$ and $\mathrm{E}_{2}(100 \mathrm{nM})$, especially by P. Among the four systems, the pGLPSAp5.8-PC3/AR system showed the least responsiveness to $\mathrm{C}, \mathrm{P}$ and $\mathrm{E}_{2}$.

Responsiveness of the four luciferase expression systems to BaP We then evaluated the responsiveness of the four systems to $\mathrm{BaP}$, an AhR agonist. In this experiment, the cells were treated with $1 \mu \mathrm{M} \mathrm{BaP}$ in the presence of DHT. The DHT concentration was set at $5 \mathrm{pM}$ for the pGLPSAp5.8-PC3/AR 

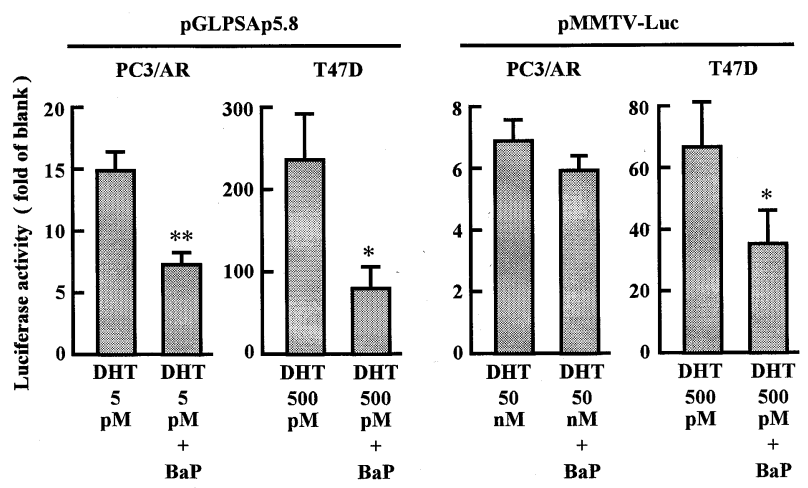

Fig. 3 Responsiveness of the four androgen-responsive luciferase expressing systems to $\mathrm{BaP}$. Concentration of $\mathrm{BaP}, 1 \mu \mathrm{M}$. Each column and vertical bar represent the mean and SD, respectively, from five separate cultures. * and **: Significantly different from corresponding DHT alone treatment $(p<0.05$ and $p<0.01$, respectively).

system, $500 \mathrm{pM}$ for the pGLPSAp5.8-T47D system, $50 \mathrm{pM}$ for the pMMTV-Luc-PC3/AR system or $500 \mathrm{pM}$ for the pMMTVLuc-T47D system, which gives approximately $50 \%$ of the maximum response shown in Fig. 1 in each luciferase expressing system. As shown in Fig. 3, BaP reduced the DHTinduced luciferase activity in the systems, except for the pMMTV-Luc-PC3/AR system. The effect of BaP was concentration-dependent in the three systems (data not shown). The three systems were found to be capable of responding to chemicals with AhR agonist activity, such as BaP.

Together these findings indicate that the pGLPSAp5.8-PC3/AR system is the most suitable among the four androgen-responsive luciferase expressing systems in terms of the sensitivity and selectivity to androgen and the responsiveness to the AhR agonist.

Responsiveness of the pGLPSAp5.8-PC3/AR system to synthetic $A R$ agonist and antagonists

Then, we further examined the responsiveness of the pGLPSAp5.8-PC3/AR system to R1881, a synthetic AR agonist, and B and CPA, synthetic AR antagonists. R1881 induced luciferase in a concentration-dependent manner in both the absence and presence of 5 pM DHT; the effect of R1881 was additive to that of DHT (Fig. 4-A). While BCT, a nonsteroidal AR agonist, and CPA, a steroidal AR antagonist, did not induce luciferase at concentrations below $10 \mu \mathrm{M}$ in the absence of DHT, they inhibited the DHT-induced luciferase activity in the presence of $5 \mathrm{pM}$ DHT in a concentrationdependent manner (Fig. 4-B). The pGLPSAp5.8-PC3/AR system was found to respond to synthetic $A R$ agonists and $A R$ antagonists.

\section{AhR-mediated antiandrogenic effect in the pGLPSAp5.8-PC3/AR} system

$\mathrm{BaP}$ exhibited an antiandrogenic effect in the pGLPSAp5.8-PC3/AR system. Here, we examined whether AhR agonists showed their antiandrogenic effects through the action of AhR in the pGLPSAp5.8-PC3/AR system or not. Four PAHs were selected, representing AhR agonists (BkF and $\mathrm{BaP}$ ) and non-agonists (Ant and Pyr), as demonstrated in MCF7 cells. ${ }^{29,30}$ They were evaluated for their antiandrogenic effect. The obtained results are shown in Fig. 5. While Ant $(1 \mu \mathrm{M})$ and Pyr $(1 \mu \mathrm{M})$ did not elicit any significant antiandrogenic effect, $\mathrm{BkF}(1 \mu \mathrm{M})$ and $\mathrm{BaP}(1 \mu \mathrm{M})$ exerted significant antiandrogenic

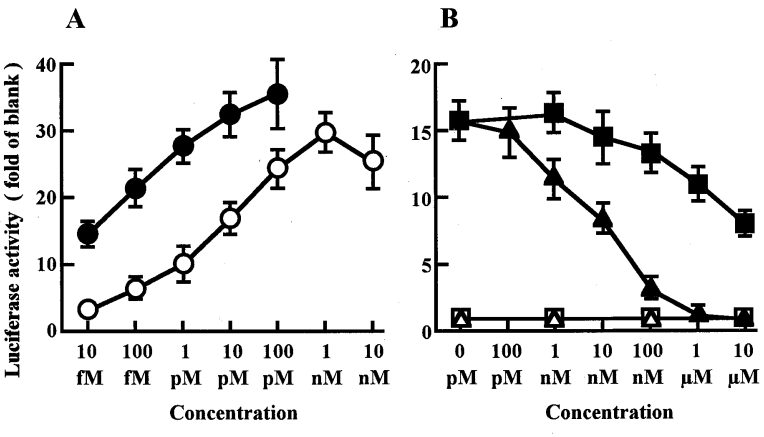

Fig. 4 Responsiveness of the pGLPSAp5.8-PC3/AR system to R1881, BCT and CPA in the absence and presence of DHT. O, R1881 alone; •, R1881 in combination with 5 pM DHT; $\triangle$, BCT alone; $\boldsymbol{\Delta}$, BCT in combination with 5 pM DHT; $\square$, CPA alone; $\mathbf{a}$, $\mathrm{CPA}$ in combination with $5 \mathrm{pM}$ DHT. Each point and vertical bar represent the mean and $\mathrm{SD}$, respectively, from five separate cultures.

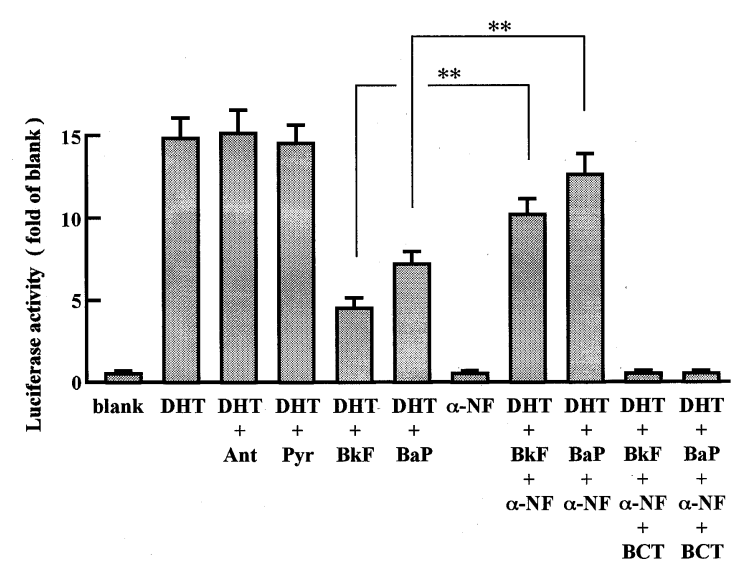

Fig. 5 Responsiveness of the pGLPSAp5.8-PC3/AR system to AhR-mediated antiandrogenic effect. Concentration: DHT, 5 pM; Ant, Pyr, BkF and BaP, $1 \mu \mathrm{M} ; \alpha-\mathrm{NF}, 1 \mu \mathrm{M}$; BCT, $10 \mu \mathrm{M}$. Each column and vertical bar represent the mean and $\mathrm{SD}$, respectively, from five separate cultures. $* *$ : Significantly different $(p<0.01)$.

effects, reducing the DHT-induced luciferase activity. $\alpha$-NF (1 $\mu \mathrm{M})$, a typical AhR antagonist, ${ }^{31}$ reversed the antiandrogen action of $\mathrm{BkF}$ and $\mathrm{BaP}$, whereas $\alpha-\mathrm{NF}$, itself, did not impact the luciferase activity, indicating that the antiandrogenic effects of $\mathrm{BkF}$ and $\mathrm{BaP}$ are mediated by activated $\mathrm{AhR}$. Further, the luciferase activity of the cells treated with DHT, BkF or BaP, and $\alpha$-NF were completely blocked by BCT $(10 \mu \mathrm{M})$, indicating that the expression of luciferase is mediated by AR. The findings shown in Fig. 5 confirmed that the pGLPSAp5.8PC3/AR system responds to AhR-mediated antiandrogenic effects.

\section{Conclusion}

The present study demonstrated that the pGLPSAp5.8-PC3/AR system is the most sensitive and selective to AR agonists among the four androgen-responsive luciferase expressing systems, and is also responsive to AR antagonists and AhR agonists. This is the first system in which the expression of luciferase is driven by the promoter region of the PSA gene, a typical human androgen-regulated gene.

Various adverse trends in male reproductive functions have 
been observed during the last decades. ${ }^{32,33}$ These trends include increasing incidence rates of testicular and prostate cancers, declining semen quality, increasing frequencies of undescended testis and hypospadias. It has been considered that environmental chemicals with androgenic or antiandrogen activity may contribute to those adverse trends. The pGLPSAp5.8-PC3/AR system is a powerful tool for the assay of the androgenic and antiandrogenic activities of chemicals and environmental samples.

\section{Acknowledgements}

This work was supported by a Grant-in-Aid for Scientific Research on Priority Areas "The Environmental Risk of Endocrine Disruptor" (No. 14042218) and Grant-in-Aid for Scientific Research (C) (No. 13672342) to R. Kizu and Grantin-Aid for Scientific Research (B) and Grant-in-Aid for the Kanazawa University 21st-Century COE Program to K. Hayakawa, from the Ministry of Education, Culture, Sports, Science and Technology of Japan.

\section{References}

1. R. J. Kavlock, G. P. Daston, C. DeRosa, P. Fenner-Crisp, L. E. Gray, Jr., S. Kaattari, G. Lucier, M. Luster, M. J. Mac, C. Maczka, R. Miller, J. Moore, R. Rolland, G. Scott, D. M. Sheehan, T. Sinks, and H. A. Tilson, Environ. Health Perspect., 1996, 104 (Suppl. 4), 715.

2. R. E. Hester and R. M. Harrison (ed.), "Endocrine Disrupting Chemicals", 1999, Royal Society of Chemistry, Cambridge.

3. C. R. Tyler, S. Jobling, and J. P. Sumpter, Crit. Rev. Toxicol., 1998, 28, 319.

4. L. E. Gray, Jr., J. Ostby, J. Furr, C. J. Wolf, C. Lambright, L. Parks, D. N. Veeramachaneni, V. Wilson, M. Price, A Hotchkiss, E. Orlando, and L. Guillette, Human Reproduct. Update, 2000, 7, 248.

5. D. W. Silversides, C. A. Price, and G. M. Cooke, Can. J. Physiol. Pharmacol., 1995, 73, 1582.

6. L. J. Guillette, Jr., D. B. Pickford, D. A. Crain, A. A. Rooney, and H. F. Percival, Gen. Comp. Endocrinol., 1996, 101,32 .

7. E. Carlsen, A. Giwercman, N. Keiding, and N. E. Skakkebaek, Br. Med. J., 1992, 305, 609.

8. S. H. Swan, E. P. Elkin, and L. Fenster, Environ Health Perspect., 1997, 105, 1228.

9. J. Lindzey, M. V. Kumar, M. Grossman, C. Young, and D. J. Tindall, Vitam. Horm., 1994, 49, 383.

10. C. A. Quigley, A. DeBille, K. B. Marschke, M. K. ElAwady, E. M. Wilson, and F. S. French, Endocr. Rev., 1995, 16, 271.

11. W. R. Kelce, C. R. Stone, S. C. Laws, L. E. Gray, J. A. Kempaainen, and E. M. Wilson, Nature, 1995, 375, 581

12. A. M. Vinggaard, E. C. Joergensen, and J. C. Larsen,
Toxicol. Appl. Pharmacol., 1999, 155, 150.

13. B. Térouanne, B. Tahiri, V. Georget, C. Belon, N. Poujol, C. Avances, F. Orio, Jr., P. Balaguer, and C. Sultan, Mol. Cell. Endocrinol., 2000, 160, 39.

14. V. S. Wilson, K. Bobseine, C. R. Lambright, and L. E. Gray, Jr., Toxicol. Sci., 2002, 66, 69.

15. M. Takeyoshi, N. Kuga, and K. Yamasaki, Arch. Toxicol., 2003, 77, 274.

16. N. R. Jana, S. Sarkar, M. Ishizuka, J. Yonemoto, C. Tohyama, and H. Sone, Biochem. Biophys. Res. Commun. 1999, 256, 462

17. A. M. Vinggaard, C. Hnida, and J. C. Larsen, Toxicology, 2000, 145,173

18. R. Kizu, K. Okamura, A. Toriba, H. Kakishima, A Mizokami, K. L. Burnstein, and K. Hayakawa, Arch. Toxicol., 2002, 77, 335.

19. A. Mizokami, A. Gotoh, H. Yamada, E. T. Keller, and T. Matsumoto, J. Urol., 2000, 164, 800.

20. P. E. de Ruiter, R. Teuwen, J. Trapman, R. Dijkema, and A. O. Brinkmann, Mol. Cell. Endocrinol., 1995, 110, R1.

21. J. L. Dai, C. A. Maiorino, P. J. Gkonos, and K. L. Burnstein, Steroids, 1996, 61, 531.

22. R. Kizu, K. Okamura, A. Toriba, A. Mizokami, K. L. Burnstein, C. M. Klinge, and K. Hayakawa, Toxicol. Sci., 2003, 76, 299.

23. G. M. Cooke, C. A. Price, and R. J. Oko, J. Steroid Biochem. Mol. Biol., 1998, 67, 347.

24. L. E. Gray, Jr., W. R. Kelce, E. Monosson, J. S. Ostby, and L. S. Birnbaum, Toxicol. Appl. Pharmacol., 1995, 131, 108.

25. B. L. Roman, R. J. Sommer, K. Shinomiya, and R. E. Peterson, Toxicol. Appl. Pharmacol., 1995, 134, 241.

26. N. H. Ing and B. W. O'Malley, "The steroid hormone receptor superfamily", in "Molecular Endocrinology, Basic Concepts and Clinical Correlations", ed. B. D. Weintraub, 1995, Raven Press, New York, 195 - 215.

27. C. Reyes-Moreno, G. Frenette, J. Boulanger, E. Lavergne, M. V. Govindan, and M. Koutsilieris, Prostate, 1995, 26 , 260.

28. C. A. Beck, N. L. Weigel, M. L. Moyer, S. K. Nordeen, and D. P. Edward, P. Natl. Acad. Sci. USA, 1993, 90, 4441.

29. K. F. Arcaro, P. W. O'Keefe, Y. Yang, W. Clayton, and J. F. Gierthy, Toxicology, 1999, 133, 115.

30. J. H. Clemons, L. M. Allan, C. H. Marvin, Z. Wu, B. E McCarry, D. W. Bryant, and T. R. Zacharewski, Environ. Sci. Technol., 1998, 32, 1853.

31. M. Merchant, V. Krishnan, and S. Safe, Toxicol. Appl. Pharmacol., 1993, 120, 179.

32. G. Sandblom and E. Varenhorse, Biomed. Pharmacother., 2001, 55, 135.

33. J. Toppari, J. C. Larsen, P. Christiansen, A. Giwercman, P. Grandjean, L. J. Guillette Jr., B. Jégou, T. K. Jensen, P. Jouannet, N. Keiding, H. Leffers, J. A. McLachlan, O. Meyer, J. Müller, E. R.-De Meyts, T. Scheike, R. Sharpe, J. Sumpter, and N. E. Skakkebæk, Environ. Health Perspect., 1996, 104 (Suppl. 4), 741 